\title{
A Review on Forwarding Strategies in NDN based Vehicular Networks
}

\author{
Dependra Dhakal, Arpan Gautam, Sudipta Dey, Kalpana Sharma
}

Sikkim Manipal Institute of Technology, Rangpo, Sikkim, India

E-mail: arpan_202027002@smit.smu.edu.in,sdptd20@gmail.com, kalpana.s@smit.smu.edu.in

Correspondence Author: dependra.d@smit.smu.edu.in

Received September 13, 2021; Revised October 15, 2021; Accepted November 16, 2021

\begin{abstract}
Named Data Networking (NDN) is a model that has been proposed by many researchers to alter the long-established IP based networking model. It derives the content centric approach rather than host-based approach. This is gaining even more traction in the wireless network and is able to replace the conventional IP-based networking. Up to now, NDN has proven to be fruitful when used with certain limitations in vehicular networks. Vehicular networks deal with exchanging information across fast moving complex vehicle network topology. The sending and receiving of information in such a scenario acts as a challenge and thus requires an effective forwarding strategy to address this problem. Different research work has provided with multiple forwarding strategy that solves the current problem up to some limit but further research work is still longed for to get an optimum solution. This paper provides a brief survey on current existing forwarding strategies related to vehicular networks using NDN as well as providing information on various resources and technologies used in it.
\end{abstract}

Keywords: NDN, Forwarding, Vehicular Network, Named Data Network

\section{INTRODUCTION}

Named Data Networking (NDN) has been brought as an encouraging architecture for the future computer network to impart Data/Contents in the future Internet. NDN makes a transition from traditional host addressing paradigm to addressing the content itself as shown in figure 1 . Instead of naming the end-to-end hosts, NDN allows hosts to name the appropriate data or content [1]. The general architecture of NDN maintains three data structures to communicate information namely:

i. Forwarding Information Base (FIB): The FIB of an NDN router incorporates a name prefix instead of an IP address prefix, and several interfaces for the same path. It basically is the routing table in case of traditional router. 
ii. Pending Interest Table (PIT): PIT stores all the packets of interest that are not yet consumed when forwarded. Each PIT entry tracks the name and the inbound and outbound interfaces of the Interest Packet.

iii. Content Store (CS): Temporary storage for received data packets is provided by the CS. It basically is the cache memory.

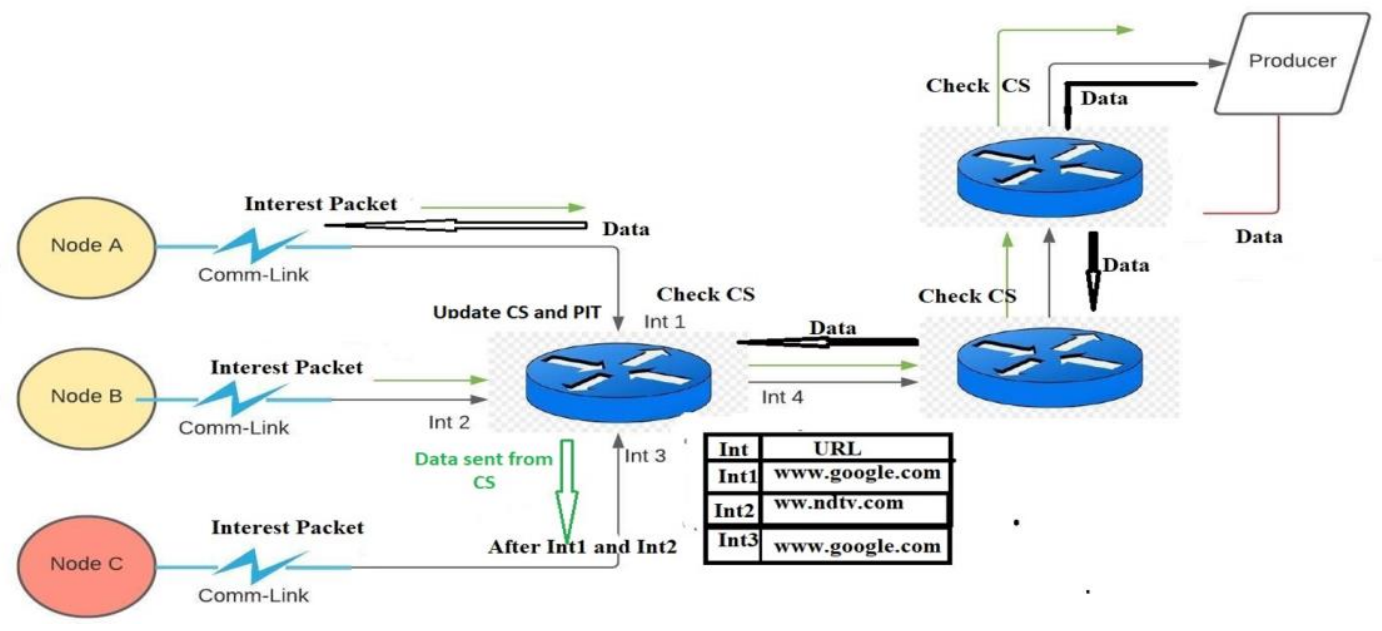

Figure 1. Working of Named Data Network

\section{RELATED WORK} follows:

Forwarding strategies according to [5] has been categorized as

i. Location Aware Forwarding: This forwarding model utilizes the geographical information of its surrounding for retrieval of Data.

ii. Context Aware Forwarding: Context aware forwarding focuses on the integrity of the information received during its communication.

iii. Neighbor Aware Forwarding: Like its name it acts according to the status of its neighbouring nodes, it basically utilizes the information of its neighbouring nodes and selects the forwarding node accordingly.

iv. Distance Aware Forwarding: It selects the forwarder nodes by calculating the distance among every neighbouring nodes.

v. Forwarding based of Link Stability: It emphasizes on the durability of the node and the strongest link of the forwarding path.

vi. Flooding Based Forwarding: It basically floods its request through most of the available neighbouring nodes.

vii. Hybrid Based Forwarding: It combines two or more type of above forwarding scheme.

\subsection{Location Aware Forwarding}

Geographical Opportunistic Forwarding Strategy (GOFS) [6] derives the "store-carry-and forward" model from the architecture of Delay Tolerant Networks (DTNs) and combines it with NDN to take benefits from the two i.e. multisource routing and router caching, the combination helps in improving the quality of Data delivery. The use of geographical location of the Position 
of Interest (POI) and the trajectories of vehicles helps in selection of another prominent relay nodes helps to enhance the communication model.

Geo-based Forwarding [7] is proposed as a reliable and efficient content delivery strategy for VANET scenarios in urban areas. Each node exchanges its information with its neighbour and vice versa, so the node requesting the data knows the exact location of the neighbour and the position of data provider for a particular Interest is also known. The forwarding strategy is categorized into forwarder selection and forwarding decision. Along with the help of naming scheme Interest can be efficiently satisfied by selecting a prominent next hop forwarder. Sometimes the selected forwarder might not be able to receive the Interest as the network topology in vehicular network is dynamic in nature and the path link previously established might break due to high speed mobility of vehicles. In such case, if the node that has not been selected as a forwarder receives the Interest, it takes the forwarding decision accordingly thus making this forwarding strategy a reliable one.

Navigo [8] aims to mitigate the problem related to constant disruption in connectivity and frequent change in the network topology of vehicles. It provides a new idea to retrieve Data packet i.e. rather than collecting the Data from a single producer it tries to fetch different chunks of that data from multiple potential providers. It does not only depend on the end Data carrier but fetches Data even from Road Side Units (RSUs) or any relay node the has the chunk cached in it. Navigo possesses a feature of self-learning the geographic location of the content providers and forwards Interests to those content carriers thus making the fetching of data faster and easier.

In order to improve efficiency and reliability in communication of information Hybrid Vehicular Name Data Networking (HVNDN) [9] integrates Interest and Data packets with acknowledgement and retransmission mechanism. HVNDN is a hybrid protocol which presents distinctive forwarding strategy, for both locations independent and location dependent contents, which are probabilistic and opportunistic in nature. The Interest packets in case of location dependent forwarding includes a field that contain the location coordinate of the required data which is not the case in location independent forwarding. HVNDN then broadcasts packet in the network.

The work proposed in [10] provides a lightweight and topology independent protocol: Reliable Forwarding Strategy (RFS). In this strategy the Data transmission mechanism is same as that of traditional Vehicular Named Data Network (VNDN) architecture i.e. Data is sent in the opposite direction of the path through which the Interest packet arrives. Since the mobility of vehicles in such networks is very high thus RFS wastes no time in forwarding the Interest packet to the correct location and also ensures the path through which the Interest has travelled is not broken. RFS makes some smart decisions before creating a link for data forwarding. It keeps track of the relative speed of the two vehicles, its distance, the direction of movement 
of the pair and checks for the direction towards which the data is to be forwarded. Depending on such factors it decides whether link creation is possible or not. If two vehicles have a huge distance gap, then such pair is least likely to be selected for link creation.

A model known as location-based and information-centric (LoICen) architecture is proposed to support the issue of broadcasting storms and improve the content requesting process [12]. This strategy separates itself from the rest as it tends to provide lesser network overhead as it opportunistically obtains the provider's position details. Though it is a location-aware forwarding technique, it does not need the data provider's details during its content search process. LoICen follows two type of process for content discovery: (i) Agnostic content discovery: This model is followed the requester node is totally unaware of the sources of the producer of content. (ii) Location oriented content discovery: This process is followed whenever the requester node knows the position of the vehicle that contains the requested data. It uses content location table (CLT) to store the location information of the node from with the data was received recently. The data provider's location information is obtained from the data packet itself, as the packet contains a cache to store the provider's location.

\subsection{Context Aware Forwarding}

Context Aware Routing Protocol CARP [11] is proposed to ensure communication of trusted information among vehicles. It aims at delivering high priority information in a much reliable and secure manner. The protocol not only contain norms to probe the trustworthiness of message but also supports NDN daemon. The protocol is encapsulated with both NDN forwarding strategies and NDN architecture. CARP is proposed to be a highly efficient routing protocol thereby improving the overall Quality of Service (QoS).

CODIE is a forwarding strategy proposed in [12] which considers propagation of data in a controlled manner. It contains a hop counter that is incremented by every node encountered until it reaches the actual provider which at last increments the counter by one and stores the value in the Data Dissemination List (DDL) field. DDL field specifies the limit up to which the data can be disseminated. The Data Dissemination Field has two benefits:

i. Restricts additional copies of data.

ii. Ensures the resultant packet to be delivered does not go beyond the actual consumer.

CODIE aims at improving the general network performance of the network by controlling the flooding of data packets. When a node that is not a potential receiver receives the interest, it increments the hop counter by one and stores the information in the PIT and then forwards it. After the interest is received by the potential receiver the hop counter is incremented and the value is stored in the DDL field. The required packet is then backtracked to the consumer node. 
CONET [13] is a controlled data propagation algorithm which aims at coping up with the problems like wastage of transmitting bandwidth, congestion caused by data packets, since they are much larger than the interest packets as they carry genuine content, and the traversal of additional copies of data. Like CODIE, CONET also has a hop counter that keeps the count of the number of hops passed by the interest packet. It also contains the Time To Live field which stores the current value of hop counter and is implanted to the data packet by the potential provider. The Time to Live (TTL) field has two advantages:

i. It secures the data packet from traversing beyond the genuine consumer.

ii. It restricts the production of auxiliary duplicates of the content.

In this forwarding strategy the Interest is forwarded with the general fields of VNDN along with an additional field to count the number of hops. When the Interest packet is received by a node, which is not a potential provider, it increments the hop counter field by one and stores its information in the PIT and forwards it further. The Interest packet on being received by the potential provider adds the current value of the hop counter in the TTL field and transfers the data packet to the sink node with the TTL field attached to it.

\subsection{Link Stability Based Forwarding}

LISF [14] is an abbreviation for Link Stability Based Interest Forwarding which minimizes the number of Interest and Data packets by predetermining the duration of link and helps relaying node to decide whether to relay the interest packet further or not. Despite making a very little improvisation on the actual VNDN architecture it maintains a good interest satisfaction rate. The work [14] claims that LISF makes the most out of the network by reducing the flooding of Interest packet and is a simple Interest forwarding methodology. LISF utilizes the mobility information disseminated by the consumer and producer vehicles. It then decides the probable relay node, which upon receiving the Interest packet, has a higher chance of recovering the required data and giving it back to the requesting vehicular node.

Link stability-based Interest forwarding for content request protocol (LISIC) [15] uses a defer timer feature that prioritizes the vehicular node when transmitting the Interest packet. It coordinates the transmission of Interest with the neighbouring nodes and thus conquers the problem of broadcasting storm caused during Interest transmission. LISIC first calculates the stability of the links. The links are then prioritized according to its stability value i.e. more stable links are likely to be kept in higher priority order. Interests are forwarded using the defer timer function which suggests that when an Interest transmission by a higher priority vehicular node is heard by low priority nodes which is transmitting the same Interest packet, 
the low priority nodes cancels its transmission. This reduces the multiple copies of Interest transmitted and the transmitting channels bandwidth is utilized efficiently.

\subsection{Neighbour Aware Forwarding}

Robust Forwarder Selection (RUFS) [16] resolves the problem of broadcast storm which occurs during various scenarios in VANETs including the communication among vehicles. It reduces the flooding of Interest by sending its Interest packet to only one neighbour node which acts as a relay node, it also mitigates the overhead of maintaining unnecessary PIT entries throughout the network. RUFS selects only one concurrent neighbour as a relay node to forward its Interest packets and thus the remaining neighbour is free from forwarding of Interest along with the additional operations related to it. This strategy does not wait for acknowledgement and selects just one concurrent neighbour for transmission of Interest packets. To keep track of the interest satisfaction rate of the selected node, individual vehicle maintains a list known as Neighbour Satisfaction List (NSL). RUFS maintains a Recent Satisfaction List (RSL) to keep track of the Interest satisfied by the vehicle itself. In case multiple forwarders are detected then only that vehicle which has already satisfied the Interest correctly will be selected as the ultimate forwarder and rest of the neighbour discards the received Interest. Once the data Provider receives the Interest it forwards the Data back to the same path as the Interest had arrived.

Interest broadcasting storm is an important issue that needs to be considered while designing a forwarding strategy for VNDN. Distributed Interest Forwarder Selection (DIFS) strategy is proposed in [24] to resolve this issue. Here the interest packets are forwarded in both the directions i.e., forward and backward, by selecting two nodes as forwarder. This not only helps to find the data producer faster but also reaches the producer with lesser Interest flooding. A data structure called Neighbour List is maintained by all the nodes that keeps the information about the nodes at its 1-hop distance. DIFS selects the potential interest forwarder by taking three parameters into consideration.

(i) 1-hop distance

(ii) Relative speed

(iii) Link duration

On the basis of these parameters each node is provided with a weight that basically acts as priority on the basis of which the next potential forwarder is selected.

The Push-based Data Forwarding (PDF) [17] strategy classifies content into two categories: Regular content and Critical content. Regular content demands frequent updates and in order to forward such content an Interest packet is mandatory. Critical content can be produced during important events and can be generated anytime. Critical information such as safety of the driver or some misconduct by other drivers are stored by the producer 
vehicle. PDF adds a new field to the existing NDN data packet know as object size that stores name of the content and its size. The producer vehicle transmits the content chunk along with the object size field to its neighbouring vehicles, these neighbours make a temporary PIT entry upon receiving the chunk. Later on, when the neighbouring vehicles receive more chunks, it caches those chunks which matches the previous PIT entry. For enhancing the packet delivery ratio, a Density-Aware Delay-Tolerant (DADT) approach is proposed [22]. A "differing timer" is used for the purpose of eliminating broadcast storm. The value of this parameter is calculated using the location information of both the nodes and the data producers. The name of the data is appended with the values of its location coordinates and the interfaces through which the data or the interest packets travels also attaches their ID and current location information with the packets. It also maintains a neighbour list that updates itself periodically whenever a packet in-the-air is overheard. The communication phase in DADT occurs in two phases rebroadcast and re-transmission. In the former phase a deferring time is set to all the nodes that receive the request for the same interest and all the nodes are given a priority value. The node with the highest priority gets to transmit first. The advantage of inculcating this condition is that it not only avoids collision of Interest packets but also reduces flooding of Interest and apparently reduces unnecessary transmission overhead. The priorities are set considering two factors:

- The highest priority is given to the node which is farther away from the last hop, to transmit the packet faster with lesser number of hops.

- The node nearest to the data source is given highest priority.

For these purposes a reference node is selected which is closer to the data provider and is within the transmission range of the last hop. The retransmission phase is not always applicable. A separate pending retransmission table stores the interest that it has forwarded. The interest is retransmitted whenever it detects a node with forwarding capabilities. If the interest forwarded by some other node is overheard the interest in the pending retransmission list is deleted.

\subsection{Distance Aware Forwarding}

Named Data VANET Protocol (NVP) [18] ensures higher probability of packet delivery as it broadcasts the packet into the network. The broadcast feature is upgraded to provide incremental broadcast and adaptive broadcast. NVP segregates packets into two types, request packet and reply packet, and this are distinctly identified by the type file included in the packet header. Each node in NVP maintains a Transmission Cost table which stores information related to the node from which it receives the packet. Such information includes MAC address, transmission cost value between the two nodes and the current sequence number. The information related to the node's one hop neighbour is stored in Neighbour table. Responder table maintains information related to the producer that has recently satisfied its 
Interest so that the next Interest can directly be forwarded to that node instead of using broadcast strategy.

The Opportunistic Interest Forwarding Protocol (OIFP) [19] uses the inter node distance information to prioritize the transmission of Interest between neighbouring vehicles. It is a lightweight protocol that addresses the problem of unnecessary Interest packet transmission in VNDN. It calculates the Interest transmission priority for every receiver which is done by taking into consideration the location information of both neighbouring nodes and the active forwarder node. The priority is executed using defer timer i.e., the Interest transmission defer timer gets lower with the increase in distance between a node and the sender.

\subsection{Flooding Based Forwarding}

$\mathrm{N}$-Epidemic [20] is a flooding based forwarding scheme derived from an IP based data forwarding scheme (Epidemic). Here the Interest packet is dispersed by a consumer vehicle to all its neighbour which acts as a relay node. The Interest packet spreads like an epidemic until a potential receiver is encountered. The Data packet is then sent to the sink node using the same process.

$\mathrm{N}$-Spray and Wait [20] is another flooding-based scheme that is derived from an IP based forwarding scheme: Spray and Wait. A consumer vehicle selects first ' $\mathrm{N}$ ' vehicular nodes and forwards the Interest packet to them. These vehicles act as a carrier until it encounters a potential provider.

The usual IP based data forwarding scheme Adaptive has been adapted and implemented along with NDN as a forwarding scheme for vehicular networks. The N-Adaptive [20] forwarding scheme first checks the status of the Interest in another vehicle's PIT entry before forwarding the packet. The packet is handed to another vehicle only if its PIT does not contain the state of the packet else the forwarding does not take place.

\subsection{Hybrid Forwarding}

Mobility Predict Based Forwarding Strategy (MPFS) proposed in [23] aims at reducing the interest broadcasting storm. It also checks for the validity of information regarding vehicles present in the NeighBor Table (NBT). This forwarding strategy basically extracts the features of Link Stability, Neighbour-aware and Distance aware forwarding strategies.

Here a node is selected as a next-hop forwarder based on its calculated "Distance along the road (DR)", which must be at a larger distance from the current node. The next-hop forwarder is selected also based on whether it has a stable link or not, "Link Expiry Time (LET) is calculated which provides an estimation of how stable a link is. Each node updates its NBT by sharing its mobility information frequently which discards the outdated vehicle information and thereby reduces the rate of forwarding of Interest packets. 


\section{OVERVIEW OF THE PROPOSED METHOD}

Table 1 provides information about the categories, as discussed earlier in this paper, in which the corresponding forwarding strategy belongs to. The type of forwarding strategy mentioned in the Table 1 is classified based on [5]. It also provides the type of cooperative networks used. There are broadly three type of cooperative networks; Vehicle to Vehicle (V2V), Vehicle to Infrastructure (V2I) and Vehicle to Everything(V2X). The infrastructures used for communication is also known as Road side units (RSUs). The other technologies used in the algorithm for testing is also listed in the Table. Traffic simulators helps in generating real time traffic in a real time map which helps us understand the behaviour of the algorithm when simulated in such real time environments.

Some of the proposed strategies have used such simulators and are mentioned alongside them in the Table. The last column of the Table provides the density of nodes used while executing the algorithm.

During the simulation of the forwarding strategies, the vehicular nodes are incorporated with various other technologies to enable communication between themselves. Such technologies used in vehicles is listed in Table 1 alongside the forwarding strategy. It is clearly visible in Table 1 that the most commonly used technology in vehicles is IEEE 802.11p which is an extension of IEEE 802.11, the standard for Wi-Fi. The former standard is used for exchanging data between mobile vehichlar nodes. The standard is also suitable for establishing communication with RSUs. The Global Positioning System (GPS) used in some of the forwarding strategies basically provides location coordinates of the vehicles which helps in calculating the distance between the vehicular nodes and it also is useful in identifying the linking stability between two nodes. While most of technologies used during simulation are software based, some hardware tools were also used in the simulation of flooding-based strategy which is also enlisted in Table 1.

The testing of forwarding strategies under real time traffic not only helps in checking the reliability of the strategy but also helps in determining its performance. Although the deployment of the forwarding strategy in real time traffic is difficult and a tedious task, some simulation tools are available that can be used to extract the map of real time road scenario and generate traffic in the exatracted map. OpenStreetMap is an open-source map extraction tool with the help of which the map of any location in the world can be extracted. SUMO (Simulation of Urban MObility) is used to generate traffic which includes road vehicles, pedestrains and public transport. These tools are helpful in creating realtime traffic on a realtime road scenario, which helps in understanding the behaviour of the forwarding strategies. The use of such tool is listed in Table 1 coorresponding to the forwarding strategies. 
Table 1. Comparison of Different Forwarding Strategy With Type, Technology and Traffic Simulator Used and Node Density

\begin{tabular}{|c|c|c|c|c|c|c|}
\hline \begin{tabular}{|c|} 
Forwarding \\
Strategy
\end{tabular} & Type & \begin{tabular}{|c|} 
Type of \\
Cooperative \\
Network
\end{tabular} & $\begin{array}{l}\text { Technologies } \\
\text { used in Vehicle }\end{array}$ & $\begin{array}{c}\text { Traffic } \\
\text { Simulator } \\
\text { Used }\end{array}$ & $\begin{array}{l}\text { Node } \\
\text { Density }\end{array}$ & $\begin{array}{c}\text { Environment } \\
\text { condition }\end{array}$ \\
\hline GOFP & $\begin{array}{c}\text { Location } \\
\text { Aware }\end{array}$ & V2V & $\begin{array}{c}\text { GPS, wireless } \\
\text { communication } \\
\text { model (name not } \\
\text { mentioned) }\end{array}$ & none & $40-120$ & Mobile \\
\hline $\begin{array}{c}\text { Geo-based } \\
\text { Forwarding }\end{array}$ & $\begin{array}{c}\text { Location } \\
\text { Aware }\end{array}$ & V2V & Not mentioned & \begin{tabular}{|c|}
- \\
OpenStreetMap \\
-Simulation of \\
Urban Mobility \\
(SUMO)
\end{tabular} & 332 & Mobile \\
\hline NAVIGO & $\begin{array}{l}\text { Location } \\
\text { Aware }\end{array}$ & V2V, V2I & $\begin{array}{c}\text { IEEE } \\
802.11 \mathrm{p} \\
\end{array}$ & $\begin{array}{l}\text {-CORNER } \\
\text {-SUMO } \\
\end{array}$ & 812 & $\begin{array}{c}\text { Static and } \\
\text { Mobile }\end{array}$ \\
\hline HVNDN & $\begin{array}{l}\text { Location } \\
\text { Aware }\end{array}$ & $\begin{array}{c}\text { V2V (also } \\
\text { compatible } \\
\text { with } \\
\text { V2I) } \\
\end{array}$ & $\begin{array}{c}\text { GPS, sensors, IEEE } \\
802.11 \mathrm{p}\end{array}$ & -SUMO & $40-200$ & $\begin{array}{l}\text { Static and } \\
\text { Mobile }\end{array}$ \\
\hline RFS & $\begin{array}{l}\text { Location } \\
\text { Aware }\end{array}$ & V2V & IEEE WAVE, GPS & none & Random & Mobile \\
\hline LoICen & $\begin{array}{c}\text { Location } \\
\text { Aware }\end{array}$ & $\mathrm{V} 2 \mathrm{~V}$ & IEEE $802.11 p$ & - SUMO & $\begin{array}{l}200,300, \\
400 \mathrm{v}\end{array}$ & Mobile \\
\hline CODIE & $\begin{array}{l}\text { Context } \\
\text { Aware }\end{array}$ & V2V & IEEE $802.11 \mathrm{p}$ & none & $50-120$ & Mobile \\
\hline CONET & $\begin{array}{l}\text { Context } \\
\text { Aware }\end{array}$ & V2V & IEEE $802.11 p$ & none & $50-120$ & Mobile \\
\hline LSIF & $\begin{array}{c}\text { Link } \\
\text { Stability } \\
\text { Aware } \\
\end{array}$ & V2V & $\begin{array}{l}\text { IEEE } 802.11 p, \\
\text { GPS }\end{array}$ & none & $25-100$ & Mobile \\
\hline LISIC & \begin{tabular}{|c|} 
Link \\
Stability \\
Aware \\
\end{tabular} & V2V & IEEE 802.11p & -SUMO & $\begin{array}{c}30 \text { percent } \\
\text { producer } \\
\text { node }\end{array}$ & Mobile \\
\hline RUFS & $\begin{array}{c}\text { Neighbour } \\
\text { Aware }\end{array}$ & V2V & IEEE $802.11 p$ & -SUMO & $20-50$ & Mobile \\
\hline PDF & \begin{tabular}{|c|} 
Neighbour \\
Aware
\end{tabular} & V2V, V2RSU & Not mentioned & none & $\begin{array}{c}\text { not } \\
\text { mentioned }\end{array}$ & $\begin{array}{c}\text { Static and } \\
\text { Mobile }\end{array}$ \\
\hline DADT & \begin{tabular}{|c|} 
Neighbour \\
Aware
\end{tabular} & V2V, V2RSU & $\begin{array}{c}\text { Wi-Fi adhoc } \\
\text { interface, GPS }\end{array}$ & -SUMO & $20-80$ & $\begin{array}{c}\text { Static and } \\
\text { Mobile }\end{array}$ \\
\hline NVP & $\begin{array}{l}\text { Distance } \\
\text { Aware }\end{array}$ & $\mathrm{V} 2 \mathrm{~V}$ & TraCI & $\begin{array}{c}\text {-Openstreet } \\
\text { Map } \\
\text {-SUMO }\end{array}$ & $0-450$ & Mobile \\
\hline OIFP & $\begin{array}{l}\text { Distance } \\
\text { Aware }\end{array}$ & $\begin{array}{c}\text { Not } \\
\text { mentioned }\end{array}$ & Not mentioned - & SUMO & 300 & $\begin{array}{c}\text { Not } \\
\text { mentioned }\end{array}$ \\
\hline $\begin{array}{c}\text { N-Epidemic } \\
\text { and N-Spray } \\
\text { Wait and N- } \\
\text { Adaptive }\end{array}$ & $\begin{array}{l}\text { Flooding } \\
\text { Based }\end{array}$ & V2V V2R & $\begin{array}{c}\text { IEEE 802.15.4, } \\
\text { MPC5604BTRK } \\
\text { Micro-controller } \\
\text { chip, Motor drive } \\
\text { board, Servo } \\
\text { Motor, Arduino } \\
\text { Uno, Wireless } \\
\text { Shield, XBee } \\
\text { shield, XBee and } \\
\text { USB cables }\end{array}$ & none & $\begin{array}{c}\text { not } \\
\text { mentioned }\end{array}$ & $\begin{array}{l}\text { Static and } \\
\text { Mobile }\end{array}$ \\
\hline MPFS & Hybrid & $\begin{array}{c}\text { Not } \\
\text { mentioned }\end{array}$ & IEEE $802.11 \mathrm{p}$ & SUMO & $50-250$ & $\begin{array}{c}\text { Not } \\
\text { mentioned }\end{array}$ \\
\hline DIFS & $\begin{array}{c}\text { Neighbour } \\
\text { Aware }\end{array}$ & $\begin{array}{c}\text { Not } \\
\text { mentioned }\end{array}$ & Not mentioned & Not mentioned & $20-50$ & $\begin{array}{c}\text { Not } \\
\text { mentioned }\end{array}$ \\
\hline
\end{tabular}


During simulation the variability of node density plays an important role in understanding the performance of the forwarding strategy. Node density is the number of vehicular nodes used, during simulation, for communication. The nodes can further be categorized into; a) Producer nodes, b) Consumer nodes, c) Relay nodes. The producer nodes are the node carrying the required data, consumer nodes are those who request the data and relay nodes acts as a forwarder node which upon receiving the data request forwards it to potential producer or another forwarder. In a network topology any node can act as producer, consumer or forwarder, infact a particular node can act as all three depending upon the availability of the data. The number of nodes used during simulation of the forwarding strategy is listed in Table 1. There is no limitation to the number of nodes used during simulation, increase in the node density leads to the increase in simulation time. Higher node density usage during simulation is limited to the hardware requirement of the system but can be beneficial in studying the behaviour of the forwarding strategy, as it can be tested with more evaluation parameters. Simulation with lower node density decreases simulation time and can be performed with minimal hardware specifications. Although the simulation time is reduced but the performance of the forwarding strategy in real time traffic scenario cannot be evaluated, because if the strategy were to be deployed in real time environment the vehicular node density is generally high.

Column "Environnment condition" in Table 1 contains information about the movement of the nodes during simulation, whether the nodes are static or mobile. In most forwarding strategies, which considered only V2V communication the environmental condition was mobile in nature. Some forwarding strategies also considered communication with the road side units, which are static in nature, along with V2V communication. In such case the environmental condition was both static and mobile.

The information provided in Table 2 presents various simulators that are used to test the performances of the corresponding forwarding strategies. While some of the forwarding strategies were simulated using various version of Network Simulators (NS-2, NS-3) some forwarding strategies have been tested using ndnSIM which is a NS-3 based simulator explicitly used to implement NDN communication. Some of the proposed forwarding algorithms have just been tested under hardware simulators without the use of any Network simulators. Above Table also presents information about various performance matrices used to test the algorithms. A column in this Table provides the list of algorithms with which the proposed strategy is compared with. Few conclusions drawn regarding the forwarding strategies is stated in the last column of the Table. 
Table 2: Comparison of Different Forwarding Strategy with Simulator and Algorithm Used and Evaluation Matrices

\begin{tabular}{|c|c|c|c|c|}
\hline $\begin{array}{c}\text { Forwarding } \\
\text { Strategy }\end{array}$ & $\begin{array}{c}\text { Simulator } \\
\text { Used }\end{array}$ & Algorithms & Evaluation Matrices & Conclusion \\
\hline GOFP & ONE & $\begin{array}{l}\text { - First Contact } \\
\text { - P-Random }\end{array}$ & $\begin{array}{l}\text { - INTEREST packet hops } \\
\text { - DATA packet hops } \\
\text { - INTEREST packet } \\
\text { consumption delay } \\
\text { - DATA packet delivery } \\
\text { delay }\end{array}$ & $\begin{array}{l}\text { Proposed Forwarding } \\
\text { Strategy performed } \\
\text { better comparatively }\end{array}$ \\
\hline $\begin{array}{l}\text { Geo-based } \\
\text { Forwarding }\end{array}$ & ndnSIM & $\begin{array}{l}\text { - GPRS -Original } \\
\text { NDN Forwarding } \\
\text { strategy } \\
\text { - random timer- } \\
\text { based strategy }\end{array}$ & $\begin{array}{c}\text { - request success ratio } \\
\text { - delay }\end{array}$ & $\begin{array}{l}\text { suggested forwarding } \\
\text { strategy revealed } \\
\text { significant improvement } \\
\text { in request success ratio } \\
\text { and delay }\end{array}$ \\
\hline NAVIGO & ndnSIM & - GPRS & $\begin{array}{l}\text { - success rate - User } \\
\text { satisfaction } \\
\text { - V2V channel access } \\
\text { - Load on the } \\
\text { infrastructure } \\
\text { - Infrastructure offload }\end{array}$ & $\begin{array}{l}\text { NAVIGO outperforms } \\
\text { comparatively in all the } \\
\text { evaluation matrices }\end{array}$ \\
\hline HVNDN & ndnSIM & $\begin{array}{l}\text { - R-NDN for } \\
\text { location } \\
\text { dependent } \\
\text { Forwarding - } \\
\text { Flooding scheme } \\
\text { in two directions } \\
\text { for location } \\
\text { independent } \\
\text { Forwarding }\end{array}$ & $\begin{array}{l}\text { - end-to-end delay } \\
\text { - performance overhead } \\
\text { - hop count } \\
\text { - data delivery ratio }\end{array}$ & $\begin{array}{l}\text { It outperformed the } \\
\text { mentioned strategies }\end{array}$ \\
\hline RFS & $\begin{array}{l}\text { Analytical } \\
\text { model }\end{array}$ & Not Available & $\begin{array}{l}\text { - transmission delay } \\
\text { - interest packet delivery } \\
\text { rate }\end{array}$ & $\begin{array}{l}\text { It achieves its goal with } \\
\text { both decrease in } \\
\text { transmission delay and } \\
\text { increase in interest packet } \\
\text { delivery }\end{array}$ \\
\hline LoICen & $\begin{array}{l}\text { OMNET++ } \\
\text { \&Veins }\end{array}$ & $\begin{array}{c}\text {-NDN } \\
\text {-MHMP } \\
\text {-Dynamic unicast } \\
\text { protocol } \\
\text {-OIFP } \\
\text {-LISIC }\end{array}$ & $\begin{array}{l}\text { - successful content delivery } \\
\text { rate } \\
\text { - average delay for content } \\
\text { delivery } \\
\text { - average interest } \\
\text { transmission }\end{array}$ & $\begin{array}{l}\text { It performs better than } \\
\text { NDN and DU but showed } \\
\text { similar results when } \\
\text { compared to the } \\
\text { performance of other } \\
\text { strategies }\end{array}$ \\
\hline CODIE & $\begin{array}{l}\text { Network } \\
\text { Simulator } \\
\text { (NS) }\end{array}$ & - Native VNDN & $\begin{array}{c}\text { - total Copies of Data } \\
\text { Packets Processed } \\
\text { - interest Satisfaction Rate } \\
\text { (ISR) } \\
\text { - average Interest } \\
\text { satisfaction delay (ISD) }\end{array}$ & $\begin{array}{l}\text { The proposed strategy } \\
\text { shows identical ISR with } \\
\text { decreased satisfaction } \\
\text { delay }\end{array}$ \\
\hline
\end{tabular}




\begin{tabular}{|c|c|c|c|c|}
\hline CONET & NS 2.35 & $\begin{array}{l}\text { - Conventional } \\
\text { VNDN }\end{array}$ & $\begin{array}{c}\text { - Copies of Data Messages } \\
\text { Processed (CDMP) } \\
\text { - ISR } \\
\text { - ISD }\end{array}$ & $\begin{array}{c}\text { CONET achieves } \\
\text { identical Interest } \\
\text { Satisfaction Rate (ISR) as } \\
\text { of basic VNDN decreased } \\
\text { satisfaction delay and also } \\
\text { limits the copies of data }\end{array}$ \\
\hline LSIF & ndnSIM & $\begin{array}{l}\text { - Best route } \\
\text { - multicast } \\
\text { - CODIE }\end{array}$ & $\begin{array}{c}\text { - Forwarded Data Packets } \\
\text {-Interest Satisfaction Ratio } \\
\text {-Forwarded Interest Packet } \\
\text { - Round Trip } \\
\text { Time }\end{array}$ & $\begin{array}{c}\text { Performance, in } \\
\text { comparison with other } \\
\text { mentioned strategies, was } \\
\text { shown better }\end{array}$ \\
\hline LISIC & $\begin{array}{l}\text { OMNET++ } \\
\text { \&Veins }\end{array}$ & - Vanilla VNDN & $\begin{array}{l}\text {-Interest Satisfaction } \\
\text {-average number of } \\
\text { Interest transmissions } \\
\text { - average end-to-end } \\
\text { delay }\end{array}$ & $\begin{array}{c}\text { Overall content } \\
\text { delivery rate increased }\end{array}$ \\
\hline RUFS & NS 2 & $\begin{array}{l}\text { - NAIF } \\
\text { - Data Retrieval } \\
\text { - Rate based } \\
\text { forwarding } \\
\text { - Flooding based } \\
\text { CCN strategy }\end{array}$ & $\begin{array}{c}\text { - Forwarded Interest } \\
\text { Packets (FIP) } \\
\text { - Satisfied Interest Packets } \\
\text { (SIP) } \\
\text { - Avg. Interest Satisfaction } \\
\text { Delay (ISD) } \\
\text { - Avg. Number of Hopsfor } \\
\text { Content Retrieval (HCN) }\end{array}$ & $\begin{array}{l}\text { RUFS outperformed other } \\
\text { algorithms in all the } \\
\text { evaluation matrices }\end{array}$ \\
\hline PDF & ndnSIM & - Naive VNDN & $\begin{array}{c}\text { - push of Critical Content } \\
\text { - Delay }\end{array}$ & $\begin{array}{c}\text { Performance of PDF is } \\
\text { better than Naive VNDN } \\
\text { in the provided evaluation } \\
\text { matrices }\end{array}$ \\
\hline DADT & $\begin{array}{l}\text { Modified } \\
\text { ndnSIM }\end{array}$ & - DTN strategies & $\begin{array}{c}\text { - Satisfaction Ratio } \\
\text { - Normalized Transmission } \\
\text { overhead } \\
\text { - Average DTN delay }\end{array}$ & $\begin{array}{l}\text { DADT provides higher } \\
\text { satisfaction ratio while } \\
\text { achieving minimum } \\
\text { transmission overhead } \\
\text { and lower delay }\end{array}$ \\
\hline NVP & NS 3 & - AODV & $\begin{array}{c}\text { - Throughput } \\
\text { - Transmission Delay }\end{array}$ & $\begin{array}{c}\text { NVP is more suitable for } \\
\text { VNDN environment than } \\
\text { AODV }\end{array}$ \\
\hline OIFP & $\begin{array}{l}\text { OMNET++ } \\
\quad \& \\
\text { Veins }\end{array}$ & - Vanilla VNDN & $\begin{array}{l}\text { - Fraction of clients that } \\
\text { received the content } \\
\text { - Avg. number of Interest } \\
\text { transmissions for content } \\
\text { request } \\
\text { - Avg. delay for content } \\
\text { delivery }\end{array}$ & $\begin{array}{c}\text { OIFP comparatively } \\
\text { outperformed the Vanilla } \\
\text { VNDN }\end{array}$ \\
\hline
\end{tabular}




\begin{tabular}{|c|c|c|c|c|}
\hline $\begin{array}{c}\text { N-Epidemic } \\
\text { and N-Spray } \\
\text { Wait } \\
\text { and } \\
\text { N-Adaptive }\end{array}$ & $\begin{array}{l}\text { Real time } \\
\text { Hardware } \\
\text { simulation }\end{array}$ & Not Available & $\begin{array}{c}\text {-Interest Delivery Ratio } \\
\text {-Interest-Data response } \\
\text { delay - } \\
\text { Network Throughput } \\
\text {-Overhead }\end{array}$ & $\begin{array}{l}\text { The strategy showed } \\
\text { positive response towards } \\
\text { the tested parameters }\end{array}$ \\
\hline MPFS & -ndnSIM & $\begin{array}{l}\text { - basic } \\
\text { forwarding } \\
\text { strategy } \\
\text { - Distributed } \\
\text { Interest } \\
\text { Forwarder } \\
\text { strategy }\end{array}$ & $\begin{array}{c}\text { - average of number of } \\
\text { hops } \\
\text { - average of interest } \\
\text { satisfaction delay } \\
\text { - average of the forwarded } \\
\text { interest packets } \\
\text { - ratio of interest satisfied } \\
\text { to the number of interest } \\
\text { packet sent. }\end{array}$ & $\begin{array}{l}\text { Interest satisfaction } \\
\text { ratio is comparatively } \\
\text { higher while } \\
\text { maintaining lower rate } \\
\text { of forwarding Interest } \\
\text { packets. }\end{array}$ \\
\hline DIFS & - NS-2 & $\begin{array}{l}\text {-NAIF } \\
\text {-RUFS }\end{array}$ & $\begin{array}{c}\text { - average interest } \\
\text { satisfaction delay } \\
\text { - average number of hops } \\
\text { - average number of } \\
\text { Interest satisfied } \\
\text { - number of Interest } \\
\text { forwarded }\end{array}$ & $\begin{array}{l}\text { DIFS showed higher } \\
\text { number of Interest } \\
\text { satisfaction than the } \\
\text { other two. }\end{array}$ \\
\hline
\end{tabular}

\section{PERFORMANCE EVALUATION}

To understand the behaviour under different simulation conditions and to check whether the forwarding strategy fulfils the goal for which it was developed, the forwarding strategies are evaluated on the basis of different parameters. The most commonly used evaluation metrices are presented below.

- Interest packet hops: It is the number of hops traversed by the interest packet to reach the producer node.

- Data packet hops: It is the number of hops traversed by the data packet to reach the consumer node.

- Data packet delivery delay: It is the time taken by the data packet to reach the consumer node.

- Request success ratio: It is the ratio of the number of data request received to the number of requests satisfied.

- Interest packet delivery rate: Rate at which the interest packet is delivered to the producer node.

- Interest satisfaction rate: Number of interests satisfied per unit time

- Interest Satisfaction delay: Time taken to satisfy a particular interest request

The evaluation metrices used for each forwarding strategy is listed in Table 2. The contents listed in Table 3 provides a brief observation corresponding to the forwarding strategies discussed in this paper along with their year of publication. 
Table 3: Comparison of Different Forwarding Strategy with Observation and Year of publication.

\begin{tabular}{|c|c|c|c|}
\hline $\begin{array}{l}\text { Forward } \\
\text { Strategy }\end{array}$ & Year & Observation & Related work \\
\hline GOFP & 2016 & $\begin{array}{l}\text { It excludes message drops. The size of the packet used is } \\
\text { quite small. }\end{array}$ & [Liu et al. 2016] \\
\hline $\begin{array}{l}\text { Geo-based } \\
\text { Forwarding }\end{array}$ & 2015 & $\begin{array}{l}\text { It does not comply for large packet numbers in sparse } \\
\text { scenarios. }\end{array}$ & [Bian et al. 2015] \\
\hline NAVIGO & 2015 & $\begin{array}{l}\text { In the actual Wi-Fi implementation case, the proposed } \\
\text { technique is unlikely to succeed as RSUs may not be able to } \\
\text { send and receive packets from vehicles as they may not be in } \\
\text { same SSID domain. }\end{array}$ & [Grassi et al. 2015] \\
\hline HVNDN & 2015 & $\begin{array}{l}\text { It is tested only on static scenario; The proposed work } \\
\text { is not tested on city scenario with complicated } \\
\text { mobility model. }\end{array}$ & [Deng et al. 2015] \\
\hline RFS & 2016 & $\begin{array}{l}\text { The proposed strategy is only tested through analytical } \\
\text { model and is not tested using any of the simulation } \\
\text { tools }\end{array}$ & [Lin et al. 2016] \\
\hline LoICen & 2019 & This strategy is only limited to $\mathrm{V} 2 \mathrm{~V}$ communication & $\begin{array}{l}\text { [Boukerche and } \\
\text { Coutinho et al. 2019] }\end{array}$ \\
\hline CARP & 2015 & $\begin{array}{l}\text { This work does not provide any proof of testing, the } \\
\text { proposed algorithm and the analysis of performance is } \\
\text { also missing. }\end{array}$ & $\begin{array}{l}\text { [Ahmad and Adnane } \\
\text { et al. 2015] }\end{array}$ \\
\hline CODIE & 2016 & $\begin{array}{l}\text { The proposed strategy has not yet been tested as } \\
\text { feasible solution for Intelligent Transport System. }\end{array}$ & [Ahmed et al. 2016] \\
\hline CONET & 2016 & $\begin{array}{l}\text { The proposed work does not include implementation of } \\
\text { CONETin a test bed environment. }\end{array}$ & $\begin{array}{l}\text { [Ahmed and Gerla et } \\
\text { al. 2016] }\end{array}$ \\
\hline LSIF & 2018 & $\begin{array}{l}\text { LISF has not been implemented for nonlinear vehicle } \\
\text { trajectory }\end{array}$ & [de Sousa et al. 2018] \\
\hline LISIC & 2017 & $\begin{array}{l}\text { It does not solve the problem of redundant data } \\
\text { transmission }\end{array}$ & $\begin{array}{l}\text { [Boukerche et al. } \\
\text { 2017] }\end{array}$ \\
\hline RUFS & 2015 & $\begin{array}{l}\text {-RUFS has not been tested in scenarios having } \\
\text { multiple providers for any specific content } \\
\text {-relay nodes having a caching policy -multiple IEEE } \\
\text { 802.11p interfaces. }\end{array}$ & [Ahmed et al. 2015] \\
\hline $\mathrm{PDF}$ & 2016 & PDF is not tested on any VNDN scenarios. & [Majeed et al. 2016] \\
\hline DADT & 2016 & Testing using dynamic node density is not performed. & [Kuai et al. 2016] \\
\hline NVP & 2016 & $\begin{array}{l}\text { The simulation is compared with just AODV and } \\
\text { testing it with actual Forwarding Strategies of VNDN is } \\
\text { missing. }\end{array}$ & [Wang et al. 2016] \\
\hline OIFP & 2017 & Redundant data packet transmission is not handled & [Yu et al. 2017] \\
\hline $\begin{array}{l}\text { N-Epidemic } \\
\text { and N-Spray } \\
\text { Wait and } \\
\text { N-Adaptive }\end{array}$ & 2017 & $\begin{array}{l}\text { Some important aspects of Vehicular Network are not } \\
\text { approached like congestion, packet loss etc. Testing in } \\
\text { dynamic network environment is not performed }\end{array}$ & [Saxena et al. 2017] \\
\hline MPFS & 2020 & Mobility of producer node is not considered & [Wang et al. 2020] \\
\hline DIFS & 2017 & Simulation with higher node density not performed. & [Ahmed et al. 2017] \\
\hline
\end{tabular}




\section{CONCLUSION}

During the survey it was found out that the major challenge for forwarding in vehicular named data networks is to attenuate the interest broadcasting storm and deliver the requested data with minimum transmission delay. Most works proposed have majorly focused on solving these problems, while most have gained positive results while simulating in static environments but testing and incorporating it in real time scenario is still a major challenge. Although various forwarding strategies have been proposed by various authors the best one which produces optimum results and has the ability to fully be incorporated in real life vehicular environment is still awaited. This paper highlights the information regarding basic features of NDN, it also briefs on the use of NDN in vehicular networks. The main section of the paper deals with explanation of existing forwarding strategies for Vehicular Named Data Networks and also highlights the technologies used along with its outcome.

\section{REFERENCES}

[1] Saxena D, Raychoudhury V, Suri N, Becker C, Cao J. Named data networking: a survey. Computer Science Review. 2016 Feb 1;19:1555.

[2] Wang L, Afanasyev A, Kuntz R, Vuyyuru R, Wakikawa R, Zhang L. Rapid traffic information dissemination using named data. InProceedings of the 1st ACM workshop on emerging name-oriented mobile networking design-architecture, algorithms, and applications 2012 Jun 11 (pp. 7-12).

[3] Grassi G, Pesavento D, Pau G, Vuyyuru R, Wakikawa R, Zhang L. VANET via named data networking. In 2014 IEEE conference on computer communications workshops (INFOCOM WKSHPS) 2014 Apr 27 (pp. 410-415). IEEE.

[4] Shemsi I, Kadam P. Named data networking in VANET: a survey. International Journal of Scientific Engineering and Science. 2017;1(11):45-9.

[5] Tariq A, Rehman RA, Kim BS. Forwarding strategies in NDN-based wireless networks: A survey. IEEE Communications Surveys \& Tutorials. 2019 Aug 16;22(1):68-95.

[6] Liu X, Nicolau MJ, Costa A, Macedo J, Santos A. A geographic opportunistic forwarding strategy for vehicular named data networking. InIntelligent distributed computing IX 2016 (pp. 509521). Springer, Cham.

[7] Bian C, Zhao T, Li X, Yan W. Boosting named data networking for efficient packet forwarding in urban VANET scenarios. InThe 21st IEEE International Workshop on Local and Metropolitan Area Networks 2015 Apr 22 (pp. 1-6). IEEE.

[8] Grassi G, Pesavento D, Pau G, Zhang L, Fdida S. Navigo: Interest forwarding by geolocations in vehicular named data networking. 
In2015 IEEE 16th International Symposium on A World of Wireless, Mobile and Multimedia Networks (WoWMoM) 2015 Jun 14 (pp. 1-10). IEEE.

[9] Deng G, Xie X, Shi L, Li R. Hybrid information forwarding in VANETs through named data networking. In2015 IEEE 26th Annual International Symposium on Personal, Indoor, and Mobile Radio Communications (PIMRC) 2015 Aug 30 (pp. 1940-1944). IEEE.

[10] Lin Z, Kuai M, Hong X. Reliable forwarding strategy in vehicular networks using NDN. In2016 IEEE 84th Vehicular Technology Conference (VTC-Fall) 2016 Sep 18 (pp. 1-5). IEEE.

[11] Ahmad F, Adnane A. Design of trust-based context aware routing protocol in vehicular networks.

[12] Ahmed SH, Bouk SH, Yaqub MA, Kim D, Song H, Lloret J. CODIE: Controlled data and interest evaluation in vehicular named data networks. IEEE Transactions on Vehicular Technology. 2016 Apr 27;65(6):3954-63.

[13] Ahmed SH, Bouk SH, Yaqub MA, Kim D, Gerla M. CONET: Controlled data packets propagation in vehicular named data networks. In2016 13th IEEE Annual Consumer Communications \& Networking Conference (CCNC) 2016 Jan 9 (pp. 620-625). IEEE.

[14] de Sousa AM, Araújo FR, Sampaio LN. A link-stability-based interestforwarding strategy for vehicular named data networks. IEEE Internet Computing. 2018 Jun 11;22(3):16-26.

[15] Boukerche A, Coutinho RW, Yu X. Lisic: A link stability-based protocol for vehicular information-centric networks. In2017 IEEE 14th International Conference on Mobile Ad Hoc and Sensor Systems (MASS) 2017 Oct 22 (pp. 233-240). IEEE.

[16] Ahmed SH, Bouk SH, Kim D. RUFS: RobUst forwarder selection in vehicular content-centric networks. IEEE Communications Letters. 2015 Jul 1;19(9):1616-9.

[17] Majeed MF, Ahmed SH, Muhammad S, Dailey MN. Poster: PDF: Pushbased data forwarding in vehicular NDN. InProceedings of the 14th Annual International Conference on Mobile Systems, Applications, and Services Companion 2016 Jun 25 (pp. 54-54).

[18] Wang X, Liu W, Yang L, Zhang W, Peng C. A new content-centric routing protocol for vehicular ad hoc networks. In2016 22nd AsiaPacific Conference on Communications (APCC) 2016 Aug 25 (pp. 552558). IEEE.

[19] Yu X, Coutinho RW, Boukerche A, Loureiro AA. A distance-based interest forwarding protocol for vehicular information-centric networks. In2017 IEEE 28th Annual International Symposium on Personal, Indoor, and Mobile Radio Communications (PIMRC) 2017 Oct 8 (pp. 1-5). IEEE.

[20] Saxena D, Raychoudhury V, Becker C. Implementation and performance evaluation of name-based forwarding schemes in V- 
NDN. InProceedings of the 18th International Conference on Distributed Computing and Networking 2017 Jan 5 (pp. 1-4).

[21] Boukerche A, Coutinho RW. LoICen: A novel location-based and information-centric architecture for content distribution in vehicular networks. Ad Hoc Networks. 2019 Oct 1;93:101899.

[22] Kuai M, Hong $X, Y u$ Q. Density-aware delay-tolerant interest forwarding in vehicular named data networking. In2016 IEEE 84th Vehicular Technology Conference (VTC-Fall) 2016 Sep 18 (pp. 1-5). IEEE.

[23] Wang J, Luo J, Zhou J, Ran Y. A Mobility-Predict-based Forwarding Strategy in Vehicular Named Data Networks. InGLOBECOM 20202020 IEEE Global Communications Conference 2020 Dec 7 (pp. 01-06). IEEE.

[24] Ahmed SH, Bouk SH, Yaqub MA, Kim D, Song H. DIFS: Distributed interest forwarder selection in vehicular named data networks. IEEE Transactions on Intelligent Transportation Systems. 2017 Nov 22;19(9):3076-80. 